\title{
Comparisons of microbiological characteristics and antibiotic resistance of Klebsiella pneumoniae isolates from urban rodents, shrews, and healthy people
}

Xue-shan Zhong ${ }^{1}$, Yong-zhi Li ${ }^{1}$, Jing Ge${ }^{1}$, Gang Xiao ${ }^{2}$, Yun Mo ${ }^{1}$, Yu-qi Wen ${ }^{1}$, Jing-ping Liu², Yi-quan Xiong ${ }^{1}$, Min Qiu', Shu-ting Huo ${ }^{1}$, Ming-ji Cheng ${ }^{1}$ and Qing Chen ${ }^{1 *}$

\begin{abstract}
Background: The comparisons of molecular characterization and antibiotic resistance of Klebsiella pneumoniae (KP) isolates from humans and other animal hosts are not well studied. Our goal was to compare the molecular epidemiology of KP strains that were isolated from urban rodents, shrews, and healthy people.

Results: K. pneumoniae (KP) isolates were isolated from fecal samples of rodents, shrews and healthy adults in 2015 in southern China. In total, 465 fecal samples were collected, of which 85 from rodents, 105 from shrews, and 275 from healthy adults. Antimicrobial susceptibility and production of extended-spectrum $\beta$-lactamases (ESBL) of the isolates were tested. PCR-based methods were used to detect specific genes, including ESBL genes (bla $a_{\mathrm{TEM}}, b / a_{\mathrm{SHV}}$, and bla $a_{\mathrm{CTX}-\mathrm{M}}$ ) in ESBL-producing isolates, capsular serotypes (K1, K2, K5, K20, K54, and K57) in hypervirulent KPs (hvKPs), and virulence genes (magA, wcaG, rmpA, uge, kfu, and aerobactin) in hvKP isolates. Multilocus sequence type (MLST) and pulsed-field gel electrophoresis (PFGE) were performed to exclude the homology of these isolates. The carriage rate of KP in urban rodents and shrews (78.42\%) was higher than that in healthy adults $(66.18 \%)\left(X^{2}=8.206, P=0.004\right)$. The prevalence rates of ESBL-producing isolates among rodents, shrews, and humans were $7.94,12.79$, and 17.03\%, respectively. The positive rates of CTX-M, TEM and SHV types in ESBL-producing isolates were 29.79, 27.66, and 17.02\%, respectively. Serotype K1, K5, K20, and K57 were detected in both small mammals and humans. PFGE typing revealed thirty-six clusters. PFGE cluster A was clustered by samples of shrews and healthy adult, with a similarity of 88.4\%. MLST typing revealed thirty-eight types. ST23 and ST35 were detected in samples of shrews and healthy adults. ST37 was detected in samples of 2 rodents and a healthy adult.

Conclusions: Overlapping serotypes of hvKP were observed in both the animals and humans. The same PFGE or MLST types were also found in isolates derived humans, rodents and shrews. Therefore, urban rodents and shrews might play a certain role in the transmission of drug-resistant and hypervirulent KP.
\end{abstract}

Keywords: Urban rodent, Shrew, Klebsiella pneumoniae, Antimicrobial resistance, Hypervirulence, PFGE, MLST

\footnotetext{
* Correspondence: 18002270308@163.com

${ }^{1}$ Department of Epidemiology, School of Public Health, Southern Medical

University, Guangzhou 510515, China

Full list of author information is available at the end of the article
}

(c) The Author(s). 2020 Open Access This article is distributed under the terms of the Creative Commons Attribution 4.0 International License (http://creativecommons.org/licenses/by/4.0/), which permits unrestricted use, distribution, and reproduction in any medium, provided you give appropriate credit to the original author(s) and the source, provide a link to the Creative Commons license, and indicate if changes were made. The Creative Commons Public Domain Dedication waiver (http://creativecommons.org/publicdomain/zero/1.0/) applies to the data made available in this article, unless otherwise stated. 


\section{Background}

Klebsiella pneumoniae (KP) is ubiquitous in humans, animals, sewage, soil, and polluted waters [1]. KP is considered an important cause of community-acquired (CA) pneumonia infections. Since the early 1970s, KP has been detected in hospital environments and has become a leading cause of hospital-acquired (HA) infections, such as nosocomial pneumonia, wound, soft tissue, or urinary tract infections in neonates, the elderly, and immunodeficient patients [2-4]. Recently, life-threatening cases of $\mathrm{CA}$ infections, including pyogenic liver abscesses, meningitis, and endophthalmitis, caused by KP have been reported in the general population [5].

Efficiency of colonization and the ability to acquire resistance to antibiotics enables $\mathrm{KP}$ to spread rapidly in healthcare centers [4]. With the emergence of multidrugresistant (MDR) strains associated with hospital outbreaks, MDR KP is becoming an urgent threat to public health [6]. The main molecular mechanism of drug resistance in KP is the production of extended-spectrum $\beta$-lactamases (ESBL) [7]. In the early 1980s, ESBL were first identified in KP and Serratia marcescens strains in Europe [8]. Since then, ESBLproducing KP has become widespread to a lager extent [9]. ESBL are plasmid-mediated enzymes that hydrolyze oxyimino- $\beta$ lactam agents, such as third-generation cephalosporins and aztreonam [10]. These plasmids can also carry resistance genes to other antibiotics including fluoroquinolones, cotrimoxazole, and aminoglycosides [7].

In addition, a new type of virulent variant $\mathrm{KP}$, known as hypervirulent KP (hvKP), which is associated with community-acquired infections, has emerged worldwide over the past three decades. HvKP infects both the healthy and non-immuno-compromised people more easily, and has more significant morbidity and mortality than the "classic" KP [5]. Infections caused by KP have also been described in cases from various animals in veterinary studies, including companion animals, horses, cattle, birds, monkeys, elephants, seals, guinea pigs, and rats $[11,12]$.

Urban rodents and shrews have long share living environments with humans. These small mammals may play a role as reservoirs of causative agents for various bacterial, viral, and parasitic zoonoses because of their pervasiveness and their propensity toward close contact with humans [13]. Rats have been previously reported to carry multidrug-resistant bacteria such as Escherichia coli [14, 15], Staphylococcus aureus [16], and Salmonella spp. [17]. Additionally, some species of shrews in urban areas have been found to carry several bacterial pathogens of zoonotic diseases, such as Bartonella spp. [18] and Leptospira spp. [19]. Thus, urban rodents and shrews are important reservoirs of rodent-borne diseases in cities. Increasing urbanization and poverty have resulted in the emergence or re-emergence of rodent- associated diseases in urban areas [13]. Therefore, it is necessary to understand the prevalence and microbiological characteristics of zoonotic pathogens circulating among these small mammals in urban environments. To the best of our knowledge, there has yet to be studies on the prevalence and microbiological characteristics of KP carried by urban rodents and shrews.

The objectives of this study were to understand the carriage rate of KP in urban rodents and shrews, and to characterize antimicrobial resistance and hyper virulence of KP in these small mammals from a community in southern China. Meanwhile, KP isolates from healthy adults in the community were compared with those from small mammals. Based on these results, we assessed the potential transmission among urban rodents, shrews, and humans in a community.

\section{Results}

\section{Sample collection and bacterial isolation}

Between May and September of 2015, a total of 190 rodents and shrews, including 80 Rattus norvegicus, 3 Mus musculus, 2 Rattus flavipectus, and 105 Suncus murinus (Asian house shrews), were captured. The seasonal distribution of the capture rates was mainly in June and July (Additional file 1: Figure S1). Among the 190 small mammals, KP was isolated from 149 (78.42\%) individuals, including 63 Rattus norvegicus and 86 Asian house shrews. None of Mus musculus and Rattus flavipectus was positive for KP. There was no significant difference in KP carriage rates between Rattus norvegicus (74.12\%) and house shrews $(81.90 \%)\left(\chi^{2}=0.288, P=0.591\right)$ (Additional file 2: Table S4).

A total of 275 stool samples from healthy adults were collected during the same period. The detection rate for KP was $66.18 \%(182 / 275)$. The carriage rate of KP in the small mammals was higher than that in healthy adults (78.42\% vs. $66.18 \%, X^{2}=8.206, P=0.004$ ) (Additional file 2: Table S4).

\section{Antimicrobial susceptibility}

As shown in Table 1, the antimicrobial susceptibility patterns of $331 \mathrm{KP}$ isolates from the small mammals and healthy adults were similar. All isolates had a low level of susceptibility to penicillin and cephalosporin antibiotics. Susceptibility rates to piperacillin for rodents, shrews, and healthy adults were $6.35,13.95$, and $19.23 \%$, respectively. All isolates had low susceptibility to cefazolin, which were less than 5\%. For the third-generation cephalosporin, susceptibility rates to cefotaxime were $34.92,37.21$, and $35.16 \%$, respectively; susceptibility rates to ceftazidime were $66.67,63.95$, and $65.38 \%$, respectively. Susceptibility rates to cefepime, which is one of the fourth-generation antibiotics, were 79.37, 59.30, and $54.40 \%$ for rodents, shrews, and healthy adults, respectively. 
Table 1 Antibiotic susceptibility patterns of KP isolates from urban rodents, shrews, and healthy people in 2015

\begin{tabular}{|c|c|c|c|c|c|c|c|c|c|}
\hline \multirow[t]{2}{*}{ Antibiotics } & \multicolumn{3}{|c|}{ Rodents $(n=63)$} & \multicolumn{3}{|c|}{ Shrews $(n=86)$} & \multicolumn{3}{|c|}{ Healthy adults $(n=182)$} \\
\hline & $\mathrm{S}(\%)$ & I (\%) & $\mathrm{R}(\%)$ & $\mathrm{S}(\%)$ & I (\%) & $\mathrm{R}(\%)$ & $\mathrm{S}(\%)$ & I (\%) & $\mathrm{R}(\%)$ \\
\hline piperacillin & 6.35 & 52.38 & 41.27 & 13.95 & 48.84 & 37.21 & 19.23 & 40.66 & 40.11 \\
\hline cefazolin & 3.17 & 46.03 & 50.79 & 3.49 & 45.35 & 51.16 & 2.20 & 46.70 & 51.10 \\
\hline cefuroxime & 6.35 & 90.48 & 3.17 & 4.65 & 93.02 & 2.33 & 4.95 & 92.31 & 2.75 \\
\hline cefotetan & 0 & 3.21 & 96.79 & 1.20 & 4.70 & 94.20 & 1.58 & 2.22 & 96.20 \\
\hline cefotaxime & 34.92 & 50.79 & 14.29 & 37.21 & 39.53 & 23.26 & 35.16 & 36.81 & 28.02 \\
\hline ceftazidime & 66.67 & 25.40 & 7.94 & 63.95 & 29.07 & 6.98 & 65.38 & 23.08 & 11.54 \\
\hline cefepime & 79.37 & 19.05 & 1.59 & 59.30 & 38.37 & 2.33 & 54.40 & 44.51 & 1.10 \\
\hline aztreonam & 95.24 & 1.59 & 3.17 & 93.02 & 1.16 & 5.81 & 92.31 & 5.49 & 2.20 \\
\hline meropenem & 1.63 & 26.93 & 71.44 & 0 & 23.30 & 76.70 & 0 & 13.20 & 86.80 \\
\hline norfloxacin & 100.00 & 0.00 & 0.00 & 98.84 & 0.00 & 1.16 & 96.70 & 1.10 & 2.20 \\
\hline ciprofloxacin & 92.06 & 6.35 & 1.59 & 95.35 & 3.49 & 1.16 & 91.76 & 5.49 & 2.75 \\
\hline amikacin & 98.41 & 1.59 & 0.00 & 100.00 & 0.00 & 0.00 & 98.90 & 0.55 & 0.55 \\
\hline gentamicin & 96.83 & 0.00 & 3.17 & 95.35 & 1.16 & 3.49 & 96.15 & 0.00 & 3.85 \\
\hline chloramphenicol & 84.13 & 4.76 & 11.11 & 94.19 & 0.00 & 5.81 & 89.01 & 0.55 & 10.44 \\
\hline tetracycline & 79.37 & 3.17 & 17.46 & 84.88 & 4.65 & 10.47 & 75.82 & 1.65 & 22.53 \\
\hline trimethoprim-sulfamethoxazole & 84.13 & 1.59 & 14.29 & 84.88 & 5.81 & 9.30 & 72.53 & 13.19 & 14.29 \\
\hline amoxicillin-clavulanate & 87.30 & 9.52 & 3.17 & 88.37 & 8.14 & 3.49 & 80.22 & 18.68 & 1.10 \\
\hline
\end{tabular}

$S$ Susceptible, I Intermediate, $R$ Resistant

\section{ESBL-producing and multidrug-resistant KP}

The positive rates of ESBL-producing isolates were 7.94, 12.79 , and $17.03 \%$ in rodents, shrews, and healthy adults, respectively. The multi-drug resistance rates for rodents, shrews, and healthy adults were $49.21,36.04$, and $47.80 \%$, respectively. There was no statistically significant difference among rodents, shrews, and healthy adults (Additional file 2: Table S5).

Among all ESBL-producing isolates, the prevalence of CTX-M, TEM and SHV types were $29.78 \%$ (14/47), $27.65 \%(13 / 47)$ and $17.02 \%(8 / 47)$, respectively (Additional file 2: Table S5).

\section{Capsular serotyping and virulence genes}

As shown in Additional file 2: Table S6, 20 hvKP isolates of 6 common serotypes were identified among $331 \mathrm{KP}$ isolates. All six serotypes were detected in isolates from healthy adults. Serotype K1, K5, K20, and K57 were detected in both the small mammals and the healthy adults. Among all hypervirulent isolates, the magA gene was only present in $\mathrm{K} 1$ isolates, whereas the uge gene was detectable in all serotypes. All $\mathrm{K} 1$ isolates and some K20, K54, and K57 isolates carried the wcaG gene. The Aerobactin gene was identified among all rmpA-positive isolates. All K2 isolates lacked the $k f u$ gene while the other five serotypes were positive for the $k f u$ gene. As shown in Additional file 2: Table S6, K1, K2, K20, and K57 isolates carried more of these six virulence genes than did the $\mathrm{K} 5$ and $\mathrm{K} 54$ isolates.

\section{ESBL-producing KP fingerprinting}

Forty-seven ESBL-producing KP isolates which were no direct epidemiological association showed 36 PFGE types. PFGE type A clustered by samples of a shrew(S1644) and a healthy adult(T202-2). Whose similarity of the PFGE pattern was up to $88.4 \%$ (Fig. 1). The ESBL-producing KP isolates demonstrated 38 MLST types. ST23 was detected in samples of a shrew(S1644) and a healthy adult(T2022). Likewise, ST35 were detected in samples of a shrew(S1633) and a healthy adult(T144). ST37 belonged to samples of rodents (R148, R150) and a healthy adult(T156) (Fig. 1).

Among them, because of the isolates being not collected in an outbreak investigation of KP, PFGE profile only showed partial similarity of the sample sets. Interestingly, the similarity of isolates of T48 and T47, R148 and R243, R150 and R211 were more than 85\%, respectively (Fig. 1). But each two samples of which were the same type of host that collected during the different time period, suggesting that the potential transmission may be exist among urban rodents, shrews, and humans in a community.

\section{Discussion}

\section{Carriage prevalence}

K. pneumoniae can be asymptomatically present in the gastrointestinal tract, eyes, respiratory tract, and genitourinary tract of healthy humans [3]. In most infections with $\mathrm{KP}$, colonization in the gastrointestinal tract seems 


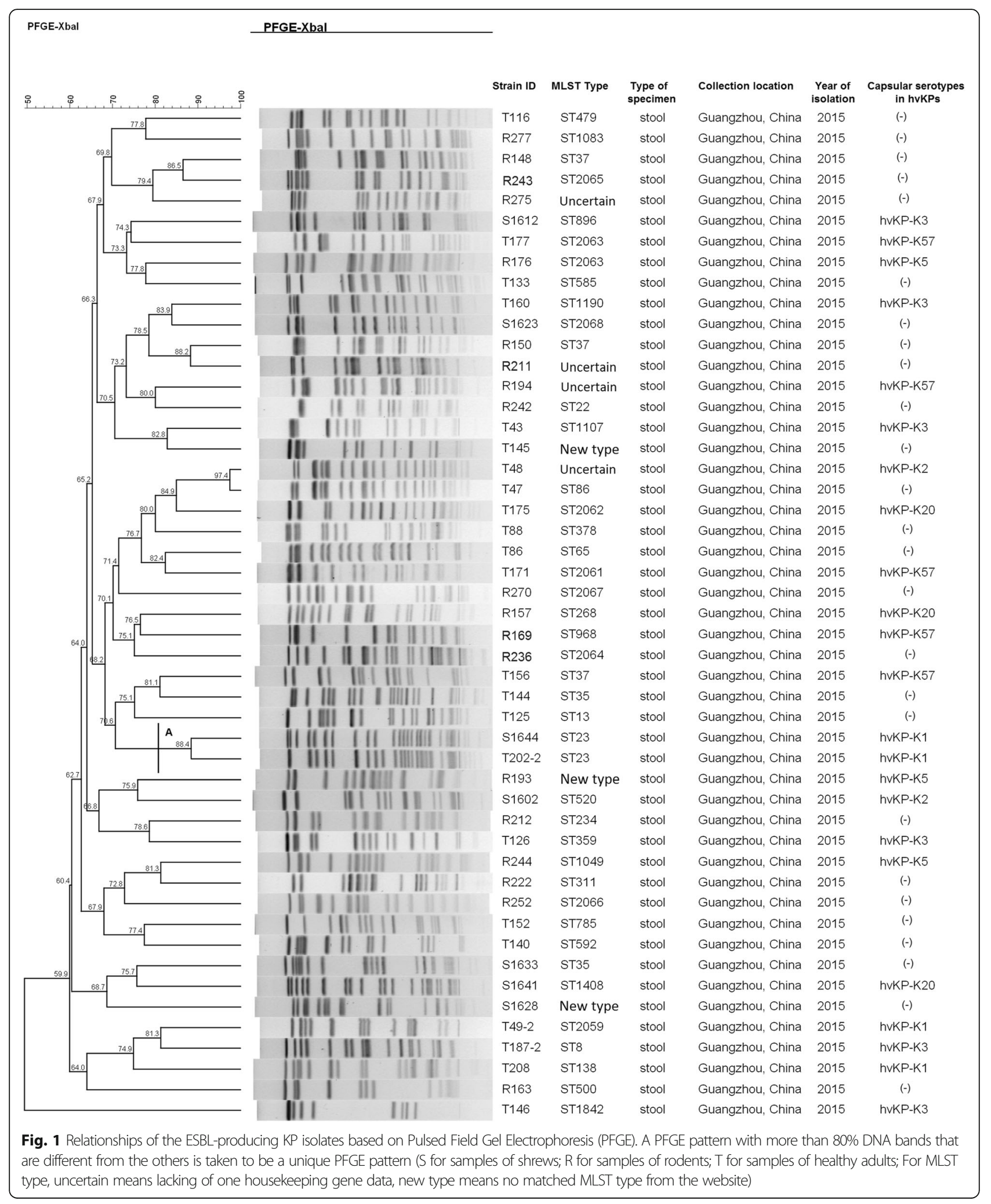

to be a prerequisite for the establishment of infections [20]. Carriage rates of KP varied considerably among different populations. In European populations, the carrier rate of $\mathrm{KP}$ in fecal samples from healthy people ranged from 10.1 to $35.7 \%$ [21, 22]. In Asian countries, the carriage rates of KP from fecal specimens varied, which was 
from 18.8 to $75.0 \%[23,24]$. In our study, the fecal carriage rate of KP from healthy adults $(66.18 \%)$ was similar to that of healthy Chinese residents in Asian countries, except Japan. Rats and shrews are known as a source of zoonotic pathogens responsible for significant human morbidity and mortality. Their feces are considered an important pathway for disseminating common bacteria through direct or indirect interactions with humans, food, water or sewage systems [13]. In our study, the fecal carriage rate of KP among these small mammals was significantly higher than that of healthy adults.

\section{Antibiotic resistance}

The emergence of KP antibiotic susceptibility, especially to third and fourth generation cephalosporins, is a critical concern for the development of treatments against bacterial infection [25]. A recent global surveillance database collected from Europe, North and South America, and Asia, showed that the detection rates for ESBLproducing KP were 7.5-44\% [26]. In China, 30.1-39.3\% of the total isolates collected from hospital or community acquired infections during 2003-2013 period were ESBL-positive [27]. In our study, we identified 7.94 and $12.79 \%$ of KP isolated from rodents and shrews to be ESBL-producers, the ESBL-positive rate among healthy adults was higher than that in studies conducted in Hungary $[28,29]$. Notably, there was no significant difference in the ESBL-positive rate among urban rodents, shrews, and healthy adults.

\section{Capsular serotypes}

Six serotypes of KP, including K1, K2, K5, K20, K54, and $\mathrm{K} 57$, are frequently considered important hypervirulent serotypes, which have been not only associated with nosocomial infections in immunocompromised patients, but also the cause of life-threatening communityacquired infections, such as liver abscess in healthy individuals, especially in Asian countries [30-32]. The results of our study showed that all six serotypes of hvKP were identified among healthy adults, and $\mathrm{K} 1, \mathrm{~K} 2$, and K57 serotypes were predominant. This finding is consistent with the previous investigation that $\mathrm{K} 1$ and $\mathrm{K} 2$ serotypes were the predominant types of hvKP among healthy Chinese in Asian countries [24]. Serotype K57 has emerged as frequent cause of septic arthritis, blood infection, urinary tract infections, and respiratory tract infection, but was rarely reported among healthy adults $[30,33]$. However, we had observed the high prevalence of $\mathrm{K} 57$ serotype isolates among healthy adults. Data on the serotype distribution of hvKP in fecal samples from urban rodents and shrews have rarely been reported previously. Among all hypervirulent isolates from these small mammals, the current study showed high prevalence of serotype $\mathrm{K} 5$ and $\mathrm{K} 57$, which were prevalent among community onset infections, including community acquired pneumonia, nasopharynx, urinary tract infection, blood infection, pyogenic liver abscess and so on [30, 34-36].

Several cases of multidrug-resistant hvKP-related hospital-acquired infections have been reported [37-39]. In our study, we found resistance to penicillin and cephalosporin antibiotics among hvKP isolates, especially in the $\mathrm{K} 5, \mathrm{~K} 20$, and $\mathrm{K} 57$ serotype isolates from healthy adults and small mammals. The antimicrobial resistance patterns of KP isolates from urban rodents, shrews, and healthy adults were similar. Furthermore, three serotypes of hvKP (K5, K20, and K57) implicated in communityacquired human infections were also detected in urban rodents and shrews.

\section{Genotypes}

Our samples were all ESBL-positive isolates, and PFGE profiles demonstrated high clonal dissemination of which in the humans and rodent animals. The higher discriminatory power of PFGE is consistent with the results of previous clinical isolates in the NICU or PICU and environmental samples [40, 41]. In our study, two isolates from shrews and healthy people, respectively, were clustered in a PFGE clustering tree. These two isolates were high-virulence of serotype K1 and ST23. The similarity of the two isolates was $88.4 \%$. In the report by Lu, et. al, ST23 was a common type of detection, which $22 \mathrm{KP}$ isolates were all from stool specimens of outpatients with diarrhea in Beijing [42]. ST23 and ST35 were detected from shrews and healthy adults in our study. In a previous study, ST37 type could be detected in the sputum and stool samples of diarrhea children [43]. ST37 genotype had the highest detection rate in rodent and human stool samples in our study. It might suggest that ST23, ST35 and ST37 KP isolates were on a wide range of hosts.

We observed that the resistance rate to cefotaxime was significantly higher than that to ceftazidime among ESBL-producing KP isolates both from small mammals and healthy adults. In analysis of ESBL genotypes, TEM, SHV, and CTX-M types were predominantly observed among KP isolates from HA- and CA-infections [25]. Researchers in China have previously identified that the predominant ESBL genotype in Beijing, Guangdong, and Hangzhou was the CTX-M type [44, 45], which preferentially exhibited powerful hydrolysis of cefotaxime compared to ceftazidime [46]. This may be a result of different hydrolysis effects with diverse ESBL genotypes.

\section{Conclusions}

To the best of our knowledge, this is the first study investigating fecal carriage of $\mathrm{KP}$ in urban rodents and shrews worldwide. It is also the first report on the 
potential relationships among KP isolates from urban rodents, shrews, and healthy adults in the community. It is meaningful to pay attention to the high prevalence and antibiotic resistance of fecal KP from urban rodents and shrews and their impact on community-acquired infections. However, due to a small size of sample in our study, bigger sample size should be needed to provide more powerful evidence in the future study.

\section{Methods}

\section{Ethics statement}

The study protocol was approved by the Animal Ethics and Welfare Committee of the School of Public Health, Southern Medical University and adhered to the guidelines for the Rules for the Implementation of Laboratory Animal Medicine (1998) from the Ministry of Health, China. All surgical procedures were performed under anesthesia with diethyl ether in efforts to minimize suffering. Endangered or protected species were not involved in this study. Healthy adults participated after signing an informed consent form. After samples were collected, the animals were sent to the animal experimental center of southern medical university for conducting harmless treatment.

\section{Collection of samples, identification and antimicrobial susceptibility testing of $K$. pneumoniae clinical isolates}

Small mammals were captured alive around houses and buildings monthly using rat-trap cages (Yue-zong Co Ltd.) in a community in Baiyun District of Guangzhou city in southern China, between May and September 2015. Some faecal samples were collected from the animals and others from healthy adults were consecutively collected in the Physical Examination Center of the third affiliated hospital of Southern Medical University in Guangzhou from May to September 2015. All stool samples were transported to the lab in transport medium at $4{ }^{\circ} \mathrm{C}$. Subsequently, stool samples were soaked in $5 \mathrm{~mL}$ of the nutrient broth (Land Bridge, Beijing, China) and incubated overnight at $37 \pm 1{ }^{\circ} \mathrm{C}$ for bacterial enrichment. The presumptive isolates were confirmed as KP using microbiological tests combined with amplification of the species-specific khe gene. Microbiological tests of the purified colonies included gram staining and biochemical testing. The khe gene was identified using PCR as described previously [47]. KP ATCC 700603 and E. coli ATCC $^{\circ} 25922$ were used as positive and negative controls, respectively, in each test protocol.

Antibiotic susceptibility testing of KP was conducted using the Kirby-Bauer disc diffusion method according to Clinical and Laboratory Standards Institute; 2018 guidelines (CLSI). A panel of 17 representative antimicrobial agents belonging to 11 different classes of antibiotics was used. Screening and phenotypic confirmatory tests for
ESBL in KP were conducted according to CLSI guidelines, 2018. Results were interpreted according to CLSI. KP (ATCC ${ }^{\circ} 700603$ ) and E. coli (ATCC ${ }^{\bullet}$ 25922) were used as quality control strains.

\section{Capsular serotyping, detection of resistance genes and hypervirulent genes}

PCR was used to detect the six common capsular serotype genes (including K1, K2, K5, K20, K54, and K57) [34, 48]. The reaction mixture was kept at $95^{\circ} \mathrm{C}$ for $3 \mathrm{~min}$, followed by 30 cycles of $94^{\circ} \mathrm{C}$ for $40 \mathrm{~s}, 58^{\circ} \mathrm{C}$ for $40 \mathrm{~s}, 72^{\circ} \mathrm{C}$ for $1 \mathrm{~min}$, and $72{ }^{\circ} \mathrm{C}$ for $7 \mathrm{~min}$. The PCR products were visualized and analyzed by agarose gel electrophoresis and sequencing. All ESBL-producing KP isolates were screened for antimicrobial resistance genes $\left(b l a_{\mathrm{TEM}}, b l a_{\mathrm{SHV}}\right.$, and $\left.b l a_{\text {СТХ-M }}\right)$ by PCR as described previously [49-51]. Virulence genes, including magA, $\operatorname{rmp} A$, uge, $k f u, w c a G$, and aerobactin among all hvKP isolates were screened by PCR using previously described methods [43, 52-56]. PCR primers and conditions have been described elsewhere (Additional file 2: Table S1, S2). All the positive products were sequenced and analyzed using the BLAST website (https://blast.ncbi.nlm.nih.gov/Blast.cgi) and the CARD website (https://card.mcmaster.ca/home).

\section{Molecular epidemiology}

Genetic relatedness among the ESBL-producing KP isolates was determined by pulsed-field gel electrophoresis (PFGE). In brief, the cell suspension of $K$. pneumoniae, placed in plugs, which were made by adding an equal volume of molten $1.0 \%$ SeaKem Gold. Slices of $K$. pneumoniae plugs were digested with $45 \mathrm{U} /$ slice $\mathrm{XbaI}$ (TaKaRa, Dalian, China) and incubated at $37^{\circ} \mathrm{C}$ for $2 \mathrm{~h}$. Electrophoresis was run on the CHEF-DRIII system $\left(120^{\circ}\right.$ angle, $6 \mathrm{~V} / \mathrm{cm}$, switch times of 6 and $\left.36 \mathrm{~s}\right)$. PFGE patterns were analyzed using the BioNumerics software package (version 5.10, Applied Maths, Inc., Austin, TX, USA). The similarity analysis was performed by Dice coefficient. A similarity of $>80 \%$ upon dendrogram analysis were considered to represent PFGE pattern subtypes [40]. A subset of isolates that represented the different PFGE clusters were further studied by multilocus sequence typing (MLST). Seven housekeeping genes (rpoB, gapA, $m d h$, pgi, phoE, infB, tonB) were detected according to the MLST official website (http://bigsdb.web.pasteur.fr/). Primer sequences, the annealing temperature, and amplified fragment sizes were shown in Additional file 2: Table S3. The reaction mixture was kept at $94{ }^{\circ} \mathrm{C}$ for $2 \mathrm{~min}$, followed by 35 cycles of $94^{\circ} \mathrm{C}$ for $20 \mathrm{~s}$, annealing for $30 \mathrm{~s}, 72{ }^{\circ} \mathrm{C}$ for $30 \mathrm{~s}$, and $72{ }^{\circ} \mathrm{C}$ for $5 \mathrm{~min}$.

\section{Statistical analysis}

Statistical analysis was carried out by using SPSS 20.0 software. The chi-square test was used to evaluate if the 
prevalence of KP was significantly different among groups. A two-sided $p$-value of $<0.05$ was considered to be statistically significant. EBURST v3.0 software was used to analyze our MLST results and database data.

\section{Supplementary information}

Supplementary information accompanies this paper at https://doi.org/10. 1186/s12866-020-1702-5.

Additional file 1: Figure S1. Tenmporal distribution of urban rodents and shrews between May and September, 2015.

Additional file 2: Table S1. Primer sequences of specific gene and resistant genes for $K$. pneumoniae. Table $\mathbf{S 2}$. Primer sequences of $K$. pneumoniae for hypenvirulent serotypes and virulent genes. Table S3. Primer sequences of seven housekeeping genes of $K$. peumoniae for multilocus sequence typing. Table S4. Carriage rates of KP isolates from urban rodents, house shrews, and healthy people. Table S5. Prevalence of multidrug resistance and ESBL-production among KP isolates from rodents, shrews, and healthy people (\%). Table S6. De tection of hypervirulent KP isolates and virulent genes from rodents, shrews, and healthy people

\section{Abbreviations}

CA: Community-acquired; CLSI: Clinical and Laboratory Standards Institute; ESBL: Extended-spectrum ß-lactamases; I: Intermediate; KP: K. pneumoniae; KP: Klebsiella pneumoniae; MDR: Multidrug-resistant; MLST: Multilocus sequence type; PCR: Polymerase-chain reaction; PFGE: Pulsed-field gel electrophoresis; R: Resistant; S: Susceptible

\section{Acknowledgements}

Grateful acknowledgement is made to my supervisor Dr. Qing Chen, who gives me considerable help by means of suggestions and comments. Her unwavering support has sustained me through frustration and depression. In addition, I deeply appreciate the contribution to this paper made in various ways by my dear friends and classmates.

\section{Disclaimers}

The opinions expressed by authors contributing to this journal do not necessarily reflect the opinions of the third affiliated hospital of Southern Medical University or the institutions with which the authors are affiliated.

\section{Authors' contributions}

XSZ analyzed and interpreted the data, and was a major contributor in writing the manuscript. QC made substantial contributions to conception and design. YZL made substantial contributions to interpreting the data and revising the manuscript. JG, YM, YQW and YQX were involved in revising the manuscript critically. GX and JPL provided technical guidance and free supply of standard bacteria. MQ, STH and MJC made substantial contributions to acquisition of data. All authors read and approved the final manuscript.

\section{Funding}

This work was supported by the National Natural Science Foundation of China (grant no. 81373051). The funding body reviewed and approved the design of the study and permitted for funding the collection, analysis of data, and the publication of our research article.

\section{Availability of data and materials}

All data generated or analyzed during this study are included in this published article and its supplementary information files. Access to raw data can be acquired by connecting to the corresponding author via email.

\section{Ethics approval and consent to participate}

The study protocol was approved by the Animal Ethics and Welfare Committee of the School of Public Health, Southern Medical University and adhered to the guidelines for the Rules for the Implementation of Laboratory Animal Medicine (1998) from the Ministry of Health, China. All surgical procedures were performed under anesthesia in efforts to minimize suffering. Endangered or protected species were not involved in this study. Healthy adults participated after signing an informed consent form.
Consent for publication

Not applicable.

\section{Competing interests}

The authors declare that they have no competing interests.

\section{Author details}

${ }^{1}$ Department of Epidemiology, School of Public Health, Southern Medical University, Guangzhou 510515, China. ²Department of clinical Laboratory, the Third Affiliated Hospital of Southern Medical University, Guangzhou 510630, China.

Received: 18 July 2019 Accepted: 9 January 2020

Published online: 14 January 2020

\section{References}

1. Bagley ST. Habitat association of Klebsiella species. Infect Control. 1985;6(2):52-8

2. Jones RN. Microbial etiologies of hospital-acquired bacterial pneumonia and ventilator-associated bacterial pneumonia. Clin infect Dis. 2010;51(Suppl 1):S81-7.

3. Podschun R, Ullmann U. Klebsiella spp. as nosocomial pathogens: epidemiology, taxonomy, typing methods, and pathogenicity factors. Clin Microbiol Rev. 1998:11(4):589-603.

4. Jarvis WR, Munn VP, Highsmith AK, Culver DH, Hughes JM. The epidemiology of nosocomial infections caused by Klebsiella pneumoniae. Infect Control. 1985;6(2):68-74.

5. Shon AS, Bajwa RP, Russo TA. Hypervirulent (hypermucoviscous) Klebsiella pneumoniae: a new and dangerous breed. Virulence. 2013;4(2):107-18.

6. Holt KE, Wertheim H, Zadoks RN, Baker S, Whitehouse CA, Dance D, Jenney A, Connor TR, Hsu LY, Severin J, et al. Genomic analysis of diversity, population structure, virulence, and antimicrobial resistance in Klebsiella pneumoniae, an urgent threat to public health. Proc Natl Acad Sci U S A. 2015;112(27):E3574-81

7. Gupta A, Ampofo K, Rubenstein D, Saiman L. Extended spectrum beta lactamase-producing Klebsiella pneumoniae infections: a review of the literature. J Perinatol. 2003;23(6):439-43.

8. Knothe $H$, Shah P, Krcmery V, Antal M, Mitsuhashi S. Transferable resistance to cefotaxime, cefoxitin, cefamandole and cefuroxime in clinical isolates of Klebsiella pneumoniae and Serratia marcescens. Infection. 1983;11(6):315-7.

9. Paterson DL, Bonomo RA. Extended-spectrum beta-lactamases: a clinical update. Clin Microbiol Rev. 2005;18(4):657-86.

10. Nathisuwan S, Burgess DS, Lewis JS 2nd. Extended-spectrum betalactamases: epidemiology, detection, and treatment. Pharmacotherapy. 2001;21(8):920-8.

11. Brisse S, Duijkeren E. Identification and antimicrobial susceptibility of 100 Klebsiella animal clinical isolates. Vet Microbiol. 2005;105(3-4):307-12.

12. Harada K, Shimizu T, Mukai Y, Kuwajima K, Sato T, Usui M, Tamura Y, Kimura $Y$, Miyamoto T, Tsuyuki Y, et al. Phenotypic and molecular characterization of antimicrobial resistance in Klebsiella spp. Isolates from Companion Animals in Japan: Clonal Dissemination of Multidrug-Resistant ExtendedSpectrum beta-Lactamase-Producing Klebsiella pneumoniae. Front Microbiol. 2016;7:1021.

13. Himsworth CG, Parsons KL, Jardine C, Patrick DM. Rats, cities, people, and pathogens: a systematic review and narrative synthesis of literature regarding the ecology of rat-associated zoonoses in urban centers. Vector Borne Zoonotic Dis. 2013:13(6):349-59.

14. Guenther S, Bethe A, Fruth A, Semmler T, Ulrich RG, Wieler LH, Ewers C. Frequent combination of antimicrobial multiresistance and extraintestinal pathogenicity in Escherichia coli isolates from urban rats (Rattus norvegicus) in Berlin, Germany. PloS one. 2012:7(11):e50331.

15. Kozak GK, Boerlin P, Janecko N, Reid-Smith RJ, Jardine C. Antimicrobial resistance in Escherichia coli isolates from swine and wild small mammals in the proximity of swine farms and in natural environments in Ontario, Canada. Appl Environ Microbiol. 2009;75(3):559-66.

16. Himsworth CG, Miller RR, Montoya V, Hoang L, Romney MG, Al-Rawahi GN, Kerr $T$, Jardine CM, Patrick DM, Tang $P$, et al. Carriage of methicillin-resistant Staphylococcus aureus by wild urban Norway rats (Rattus norvegicus). PLoS One. 2014;9(2):e87983

17. Nkogwe C, Raletobana J, Stewart-Johnson A, Suepaul S, Adesiyun A Frequency of Detection of Escherichia coli, Salmonella spp., and Campylobacter spp. in the Faeces of Wild Rats (Rattus spp.) in Trinidad and Tobago. Vet Med Int. 2011;2011:686923. 
18. Hsieh JW, Tung KC, Chen WC, Lin JW, Chien LJ, Hsu YM, Wang HC, Chomel $\mathrm{BB}$, Chang CC. Epidemiology of Bartonella infection in rodents and shrews in Taiwan. Zoonoses Public Health. 2010;57(6):439-46.

19. Mayer-Scholl A, Hammerl JA, Schmidt S, Ulrich RG, Pfeffer M, Woll D, Scholz HC, Thomas A, Nockler K. Leptospira spp. in rodents and shrews in Germany. Int J Environ Res Public Health. 2014;11(8):7562-74.

20. Montgomerie JZ. Epidemiology of Klebsiella and hospital-associated infections. Rev Infect Dis. 1979;1(5):736-53.

21. Smith GW, Blackwell CC, Nuki G. Faecal flora in spondyloarthropathy. Br J Rheumatol. 1997;36(8):850-4

22. Thom BT. Klebsiella in faeces. Lancet (London, England). 1970;2(7681):1033.

23. Chung DR, Lee H, Park MH, Jung SI, Chang HH, Kim YS, Son JS, Moon C, Kwon KT, Ryu SY, et al. Fecal carriage of serotype K1 Klebsiella pneumoniae ST23 strains closely related to liver abscess isolates in Koreans living in Korea. Eur J Clin Microbiol Infect Dis. 2012;31(4):481-6.

24. Lin YT, Siu LK, Lin JC, Chen TL, Tseng CP, Yeh KM, Chang FY, Fung CP. Seroepidemiology of Klebsiella pneumoniae colonizing the intestinal tract of healthy Chinese and overseas Chinese adults in Asian countries. BMC Microbiol. 2012;12:13.

25. Chong Y, Ito Y, Kamimura T. Genetic evolution and clinical impact in extended-spectrum beta-lactamase-producing Escherichia coli and Klebsiella pneumoniae. Infect Genet Evol. 2011;11(7):1499-504.

26. Reinert RR, Low DE, Rossi F, Zhang X, Wattal C, Dowzicky MJ. Antimicrobial susceptibility among organisms from the Asia/Pacific rim, Europe and Latin and North America collected as part of TEST and the in vitro activity of tigecycline. J Antimicrob Chemother. 2007;60(5):1018-29.

27. Yang $Q$, Zhang $H$, Wang $Y, X u$ Y, Chen M, Badal RE, Wang H, Ni Y, Yu Y, Hu $B$, et al. A 10 year surveillance for antimicrobial susceptibility of Escherichia coli and Klebsiella pneumoniae in community- and hospital-associated intraabdominal infections in China. J Med Microbiol. 2013;62(Pt 9):1343-9.

28. Ebrahimi F, Mozes J, Meszaros J, Juhasz A, Majoros L, Szarka K, Kardos G. Asymptomatic faecal carriage of ESBL producing enterobacteriaceae in Hungarian healthy individuals and in long-term care applicants: A shift towards CTX-M producers in the community. Infect Dis (London, England). 2016;48(7):557-9.

29. Ebrahimi F, Mozes J, Meszaros J, Juhasz A, Kardos G. Carriage rates and characteristics of Enterobacteriaceae producing extended-spectrum betalactamases in healthy individuals: comparison of applicants for long-term care and individuals screened for employment purposes. Chemotherapy. 2014;60(4):239-49.

30. Fung CP, Hu BS, Chang FY, Lee SC, Kuo BI, Ho M, Siu LK, Liu CY. A 5-year study of the seroepidemiology of Klebsiella pneumoniae: high prevalence of capsular serotype K1 in Taiwan and implication for vaccine efficacy. J Infect Dis. 2000;181(6):2075-9.

31. Hsu CR, Lin TL, Chen YC, Chou HC, Wang JT. The role of Klebsiella pneumoniae rmpA in capsular polysaccharide synthesis and virulence revisited. Microbiology (Reading, England). 2011;157(Pt 12):3446-57.

32. Liu YM, Li BB, Zhang YY, Zhang W, Shen H, Li H, Cao B. Clinical and molecular characteristics of emerging hypervirulent Klebsiella pneumoniae bloodstream infections in mainland China. Antimicrob Agents Chemother. 2014;58(9):5379-85

33. Lin CJ, Lin CY, Li WY, Hsiue HC, Huang YT, Ruan SY, Wang JT, Hsueh PR. Repeated bacteremia with subsequent septic arthritis caused by Klebsiella pneumoniae capsular serotype K57 in a patient with diabetes. Clin Infect Dis. 2009;49(8):1284-6.

34. Fang CT, Lai SY, Yi WC, Hsueh PR, Liu KL, Chang SC. Klebsiella pneumoniae genotype K1: an emerging pathogen that causes septic ocular or central nervous system complications from pyogenic liver abscess. Clin Infect Dis. 2007;45(3):284-93.

35. Lin WH, Wang MC, Tseng CC, Ko WC, Wu AB, Zheng PX, Wu JJ. Clinical and microbiological characteristics of Klebsiella pneumoniae isolates causing community-acquired urinary tract infections. Infection. 2010;38(6):459-64.

36. Lin YT, Wang YP, Wang FD, Fung CP. Community-onset Klebsiella pneumoniae pneumonia in Taiwan: clinical features of the disease and associated microbiological characteristics of isolates from pneumonia and nasopharynx. Front Microbiol. 2015;9:122.

37. Li W, Sun G, Yu Y, Li N, Chen M, Jin R, Jiao Y, Wu H. Increasing occurrence of antimicrobial-resistant hypervirulent (hypermucoviscous) Klebsiella pneumoniae isolates in China. Clin Infect Dis. 2014:58(2):225-32.

38. Su SC, Siu LK, Ma L, Yeh KM, Fung CP, Lin JC, Chang FY. Community-acquired liver abscess caused by serotype K1 Klebsiella pneumoniae with CTX-M-15-type extendedspectrum beta-lactamase. Antimicrob Agents Chemother. 2008;52(2):804-5.
39. Zhang Y, Zeng J, Liu W, Zhao F, Hu Z, Zhao C, Wang Q, Wang X, Chen $\mathrm{H}$, Li $\mathrm{H}$, et al. Emergence of a hypervirulent carbapenem-resistant Klebsiella pneumoniae isolate from clinical infections in China. J Infect. 2015;71(5):553-60.

40. Arhoune B, Oumokhtar B, Hmami F, Barguigua A, Timinouni M, El Fakir S, Chami F, Bouharrou A. Rectal carriage of extended-spectrum betaactamase- and carbapenemase-producing Enterobacteriaceae among hospitalised neonates in a neonatal intensive care unit in fez, Morocco. J Glob Antimicrob Resist. 2017:8:90-6.

41. Mohamed ER, Aly SA, Halby HM, Ahmed SH, Zakaria AM, El-Asheer OM. Epidemiological typing of multidrug-resistant Klebsiella pneumoniae, which causes paediatric ventilator-associated pneumonia in Egypt. J Med Microbiol. 2017;66(5):628-34.

42. Lu B, Zhou H, Zhang X, Qu M, Huang Y, Wang Q. Molecular characterization of Klebsiella pneumoniae isolates from stool specimens of outpatients in sentinel hospitals Beijing, China, 2010-2015. Gut pathogens. 2017;9:39.

43. Kitchel B, Rasheed JK, Patel JB, Srinivasan A, Navon-Venezia S, Carmeli Y, Brolund A, Giske CG. Molecular epidemiology of KPC-producing Klebsiella pneumoniae isolates in the United States: clonal expansion of multilocus sequence type 258. Antimicrob Agents Chemother. 2009;53(8):3365-70.

44. Chanawong A, M'Zali FH, Heritage J, Xiong JH, Hawkey PM. Three cefotaximases, CTX-M-9, CTX-M-13, and CTX-M-14, among Enterobacteriaceae in the People's Republic of China. Antimicrob Agents Chemother. 2002;46(3):630-7.

45. Wang H, Kelkar S, Wu W, Chen M, Quinn JP. Clinical isolates of Enterobacteriaceae producing extended-spectrum beta-lactamases: prevalence of CTX-M-3 at a hospital in China. Antimicrob Agents Chemother. 2003;47(2):790-3.

46. Rossolini GM, D'Andrea MM, Mugnaioli C. The spread of CTX-M-type extended-spectrum beta-lactamases. Clin Microbiol Infect. 2008; 14(Suppl 1):33-41.

47. Yin-Ching C, Jer-Horng S, Ching-Nan L, Ming-Chung C. Cloning of a gene encoding a unique haemolysin from Klebsiella pneumoniae and its potential use as a species-specific gene probe. Microb Pathog. 2002;33(1):1-6.

48. Turton JF, Baklan H, Siu LK, Kaufmann ME, Pitt TL. Evaluation of a multiplex PCR for detection of serotypes K1, K2 and K5 in Klebsiella sp. and comparison of isolates within these serotypes. FEMS Microbiol Lett. 2008; 284(2):247-52.

49. Grimm V, Ezaki S, Susa M, Knabbe C, Schmid RD, Bachmann TT. Use of DNA microarrays for rapid genotyping of TEM beta-lactamases that confer resistance. J Clin Microbiol. 2004;42(8):3766-74.

50. Grobner S, Linke D, Schutz W, Fladerer C, Madlung J, Autenrieth IB, Witte W, Pfeifer Y. Emergence of carbapenem-non-susceptible extended-spectrum beta-lactamase-producing Klebsiella pneumoniae isolates at the university hospital of Tubingen, Germany. J Med Microbiol. 2009;58(Pt 7):912-22.

51. Paterson DL, Hujer KM, Hujer AM, Yeiser B, Bonomo MD, Rice LB, Bonomo RA. Extended-spectrum beta-lactamases in Klebsiella pneumoniae bloodstream isolates from seven countries: dominance and widespread prevalence of SHV- and CTX-M-type beta-lactamases. Antimicrob Agents Chemother. 2003:47(11):3554-60.

52. Fang $C T$, Chuang YP, Shun CT, Chang SC, Wang JT. A novel virulence gene in Klebsiella pneumoniae strains causing primary liver abscess and septic metastatic complications. J Exp Med. 2004;199(5):697-705.

53. Nadasy KA, Domiati-Saad R, Tribble MA. Invasive Klebsiella pneumoniae syndrome in North America. Clin Infect Dis. 2007;45(3):e25-8.

54. Turton JF, Perry C, Elgohari S, Hampton CV. PCR characterization and typing of Klebsiella pneumoniae using capsular type-specific, variable number tandem repeat and virulence gene targets. J Med Microbiol. 2010:59(Pt 5):541-7.

55. Yu VL, Hansen DS, Ko WC, Sagnimeni A, Klugman KP, von Gottberg A, Goossens H, Wagener MM, Benedi VJ. Virulence characteristics of Klebsiella and clinical manifestations of K. pneumoniae bloodstream infections. Emerg Infect Dis. 2007;13(7):986-93.

56. Yu WL, Ko WC, Cheng KC, Lee CC, Lai CC, Chuang YC. Comparison of prevalence of virulence factors for Klebsiella pneumoniae liver abscesses between isolates with capsular K1/K2 and non-K1/K2 serotypes. Diagn Microbiol Infect Dis. 2008;62(1):1-6.

\section{Publisher's Note}

Springer Nature remains neutral with regard to jurisdictional claims in published maps and institutional affiliations. 